\title{
The IncRNA LOC102549805 (U1) modulates neurotoxicity of HIV-1 Tat protein
}

\author{
Bahareh Torkzaban (1)', Kalimuthusamy Natarajaseenivasan', Taha Mohseni Ahooyi ${ }^{1}$, Masoud Shekarabi', \\ Shohreh Amini', T. Dianne Langford' ${ }^{1}$ and Kamel Khalili'
}

\begin{abstract}
HIV-1 Tat is a potent neurotoxic protein that is released by HIV-1 infected cells in the brain and perturbs neuronal homeostasis, causing a broad range of neurological disorders in people living with HIV-1. Furthermore, the effects of Tat have been addressed in numerous studies to investigate the molecular events associated with neuronal cells survival and death. Here, we discovered that exposure of rat primary neurons to Tat resulted in the up-regulation of an uncharacterized long non-coding RNA (IncRNA), LOC102549805 (IncRNA-U1). Our observations showed that increased expression of InCRNA-U1 in neurons disrupts bioenergetic pathways by dysregulating homeostasis of $\mathrm{Ca}^{2+}$, mitigating mitochondrial oxygen reduction, and decreasing ATP production, all of which point mitochondrial impairment in neurons via the Tat-mediated InCRNA-U1 induction. These changes were associated with imbalances in autophagy and apoptosis pathways. Additionally, this study showed the ability of Tat to modulate expression of the neuropeptide B/W receptor 1 (NPBWR1) gene via up-regulation of IncRNA-U1. Collectively, our results identified Tat-mediated IncRNA-U1 upregulation resulting in disruption of neuronal homeostasis.
\end{abstract}

\section{Introduction}

Despite successful treatment with combination antiretroviral therapies (cART), human immunodeficiency virus type 1 (HIV-1) infection remains a persistent challenge for health care systems. In particular, even in people with HIV $(\mathrm{PWH})$ taking combination anti-retroviral therapy (cART), HIV-associated neurocognitive disorder (HAND) occurs in $\sim 30 \%$ of PWH and is a growing concern with the $\mathrm{HIV}^{+}$aging populaiton ${ }^{1-5}$. Due to its capacity to increase the production of inflammatory factors, alter gene expression, and impair of a variety of crucial signaling cascades, the effects of HIV-1 transactivator of transcription (Tat) protein on neuronal homeostasis have been subject to increasing examination ${ }^{6-12}$. For instance, some studies have documented the Tat's capacity to induce acute alterations in $\mathrm{Ca}^{2+}$ flux, leading to mitochondrial production of

\footnotetext{
Correspondence: Kamel Khalili (kamel.khalili@temple.edu)

'Department of Neuroscience, Center for Neurovirology, Lewis Katz School

of Medicine at Temple University, 3500 North Broad Street, Philadelphia, PA 19140, USA

Edited by B. Rotblat
}

reactive oxygen species $(\mathrm{ROS})^{1,11,13,14}$. Along with impairments to neuronal homeostasis, Tat interferes with the host gene expression by interacting with regulatory proteins and promoter binding factors, such as RNA Polymerase $\mathrm{II}^{15,16}$. However, the mechanistic of Tat effects on host cell gene expression and the functional consequences of such effects remain largely uncharacterized.

Among different proposed mechanisms, some studies have demonstrated various long non-coding RNAs (lncRNAs) to mediate an interplay between the host gene regulation machinery and HIV expression regulatory network $^{17-20}$. Unlike short non-coding RNAs, lncRNAs are a class of RNAs with epigenetic function to regulate gene expression at the transcriptional rather than the post-transcriptional level ${ }^{21}$. Moreover, it has been demonstrated that expression profiles of IncRNAs encoded by host cells can be altered upon viral infection ${ }^{22}$. Specifically, some recent studies signify the role of lncRNAs in the regulatory network that control HIV genome expression and replication, as well as pathogenesis and disease progression ${ }^{21,23-28}$. Moreover, several 
reports have revealed that dysregulation of IncRNAs plays a significant role in the development and progression of a variety of neurodegenerative diseases, including Alzheimer's disease (AD), Parkinson's disease (PD), Huntington's disease (HD), multiple system atrophy (MSA) and amyotrophic lateral sclerosis (ALS) ${ }^{21,29,30}$.

The current research investigated the capacity of Tat to alter host cell gene expression, particularly lncRNAs, as well as the functional consequences of such alterations on neuronal homeostasis. Utilizing a combination of molecular, cellular and genetic approaches, our results demonstrated Tat-mediated upregulation of lncRNA LOC102549805 (lncRNA-U1), along with its splicing variant (lncRNA-U1 $\left.{ }_{66}\right)$. Increased lncRNA-U1 expression was associated with the co-expression of a nearby gene NPBWR1 (Neuropeptide $\mathrm{B} / \mathrm{W}$ receptor 1 ), that encodes a transmembrane receptor involved in efficient energy expenditure $^{31,32}$. Subsequent experiments demonstrated a significant association between the upregulation of lncRNA-U1 and the dysregulation of several homeostatic pathways in primary neurons, as evidenced by increased cytosolic $\mathrm{Ca}^{2+}$, the generation of ROS by mitochondria, deficient mitochondrial membrane potential, and decreased ATP production. Moreover, immunoassays confirmed the activation of both autophagy and caspase- 3 dependent apoptotic pathways, further illustrating the consequences of Tat-mediated alterations in IncRNA-U1.

\section{Results}

\section{HIV-1 Tat alters expression of IncRNAs in neurons}

To assess the effects of HIV-1 Tat on lncRNA expression, we performed RNA-seq analysis on primary neurons derived from embryonic rat (E18) hippocampal tissue as previously described ${ }^{33}$. Among differentially expressed lncRNAs, those with transcript lengths of greater than 1000 base pairs and above two-fold change in expression (up/down-regulated) were selected for validation.

RT-PCR and qRT-PCR analyses were conducted using primers designed to validate the result of RNA-Seq and to confirm the Tat-mediated differential expression of the selected list of lncRNAs in neurons (data not shown). Of the altered IncRNAs, we focused on LOC102549805 (lncRNA-U1) that showed two to four-fold upregulation in response to Tat.

\section{Tat induces the long non-coding RNA, LOC102549805, and a novel splicing variant}

The previously uncharacterized lncRNA LOC102549805 (lncRNA-U1), located on chromosome $5 \mathrm{q} 12$ of the rat genome was identified utilizing RGD (RGD: 7711861), as well as NCBI databases ${ }^{34}$. The full-length genomic sequence and established full-length transcript of lncRNA$\mathrm{U} 1$ are $5038 \mathrm{bps}$ and $2104 \mathrm{bps}$, respectively, and include three exons with 1703, 112, and 288 bps (Fig. 1a top panel,
Supplementary Doc.1). Results from RT-PCR using a specific primer set indicated the expression of a minor splice variant for lncRNA-U1 in neurons exposed to either soluble Tat protein $(50 \mathrm{ng} / \mathrm{ml})$ or transfected with Adeno-Tat (Fig. 1b and Supplementary Fig. 2). Both amplified fragments using lncRNA-U1 forward (U1F) and reverse primers (U1Rev) were purified from the agarose gel, cloned, and sequenced to verify their specificity. Sequencing confirmed the identity of the amplified fragments and revealed that the novel splice variant contained an additional $66 \mathrm{bps}$ (Fig. 1a bottom panel and Fig. 1b). This variant was referred to as $\mathrm{U}_{66}$, provided that it differs from the identified lncRNA-U1 by $66 \mathrm{bps}^{34}$. Analysis utilizing NCBI's Basic Local Alignment Search Tool (BLAST) revealed that the extra $66 \mathrm{bps}$ constitute the intronic sequence in the immediate $5^{\prime}$ end of the second exon (Fig. 1a bottom panel, Supplementary Doc.1).

To verify the Tat-mediated alteration of IncRNA-U1, a set of exon-specific forward primers were designed to assess the expression of IncRNA-U1 isoforms in rat neurons exposed to Tat (Fig. 1c). In order to accurately measure both lncRNA-U1 and lncRNA-U1 $1_{66}$, two different forward primers (U1-1, U1-4) with an identical reverse primer were chosen for expression quantification (Fig. 1c). The U1-1 forward primer quantifies the expression level of the $\mathrm{U} 1_{66}$ isoform by amplifying the target IncRNA from the exon junction between the first exon and the additional $66 \mathrm{bps}$ $\left(\mathrm{U} 1_{66}\right)$ added to $5^{\prime}$ of the second exon and the last exon. The U1-4 primer was designed to quantify lncRNA-U1 expression, regardless of the different splice variants. qRTPCR indicated that neurons transduced with Ad-Tat demonstrated an increased expression of IncRNA-U1, and/or $\mathrm{U} 1_{66}$ by $\sim 4$-folds (Fig. 1d). Remarkably, the expression of the $\mathrm{U} 1_{66}$ isoform was nearly undetectable compared to lncRNA-U1 in control samples (i.e. untreated neurons or Ad-Null transduced neurons) (Fig. 1e). These results demonstrated the capacity of Tat to induce elevated expression, as well as splicing variation, for the target lncRNA-U1 (Fig. 1).

To further investigate variation in lncRNA-U1, we examined its stability following transcriptional inhibition and its expression in response to various Tat mutants. Specifically, we first measured lncRNA-U1 expression in Ad-Tat transduced neurons following transcriptional inhibition with actinomycin D $(2 \mu \mathrm{M})$. Following $1.5 \mathrm{~h}$ of treatment, qPCR revealed lncRNA-U1 levels in untreated neurons was reduced by $50 \%$. Conversely, neurons expressing Tat increased lncRNA-U1 expression up to $50 \%$ after $2 \mathrm{~h}$, followed by a decline observed at $4 \mathrm{~h}$ posttreatment, suggesting that Tat not only overexpressed lncRNa-U1, but also stabilized it (Fig. 1f). To identify the specificity of Tat mutants in up-regulating or stabilizing lncRNA-U1, Hek-293 cells were transfected with different Tat mutant constructs (i.e. Tat-36, Tat-72, and Tat-86) 


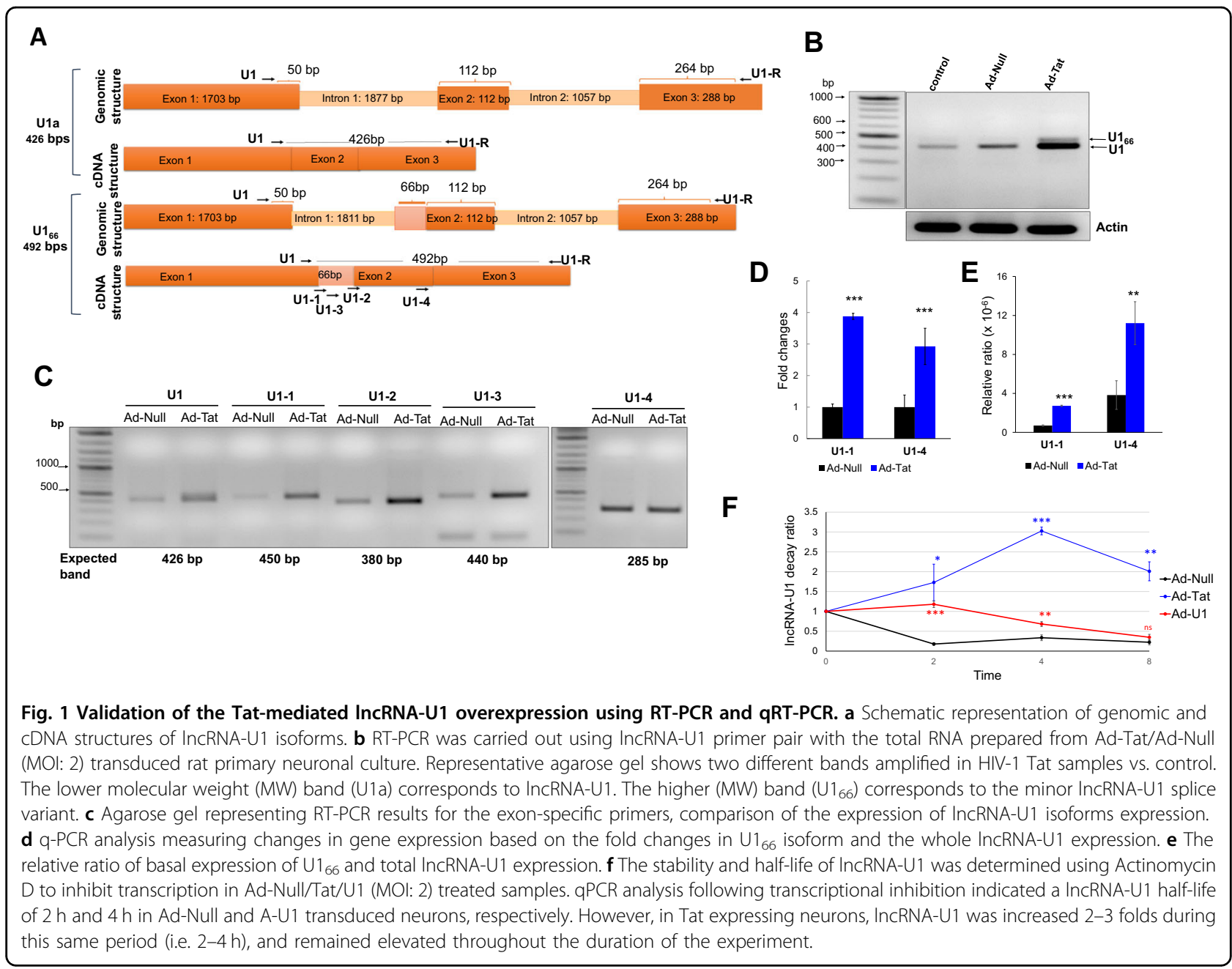

and IncRNAU1 levels were subsequently measured by qPCR. While Tat-36 and Tat-72 did not induce lncRNAU1 expression, Tat-86 significantly increased lncRNA-U1 comparable to Ad-Tat (Tat 101) (Supplementary Fig. 2b).

\section{IncRNA-U1 is a cis-regulator of NPBWR1}

Numerous studies have reported that cis-regulatory effects of lncRNAs impact transcription of nearby genes $^{29,35-38}$. In this study, we reviewed all recorded genes for quantitative trait loci (QTLs) in the 5q region of the rat genome (RGD:7711861) to assess the potential influence of lncRNA-U1 on nearby genes. QTLs are the genomic regions mapped by identifying a correlation between molecular elements and observed traits ${ }^{39-41}$. Thus, QTL studies usually indicate the association between genetic markers and a target phenotype, which is relevant in pathological studies. Therefore, the list of the genes belonging to QTLs on chromosome $5 \mathrm{q}$ (i.e. the location of lncRNA-U1) of the rat genome was compared to the list of differentially expressed genes identified via RNA-seq (Fig. S2). In turn, the neighboring $N P B W R 1$ (Neuropeptide $\mathrm{B} / \mathrm{W}$ receptor 1 ) gene was found to be upregulated by greater than twofold in response to Tat exposure. NPBWR1 with a full transcript of 990 bps was located about $211 \mathrm{kbp}$ downstream from the IncRNA-U1 sequence and was the closest coding gene in the lncRNA-U1 vicinity. The result of qRT-PCR analysis confirmed the increased expression of NPBWR1 when neurons were exposed to either soluble Tat protein or transduced with Ad-Tat for $72 \mathrm{~h}$ (Fig. 2a). Western blot analysis validated the significant upregulation of NPBWR1 upon Tat expression in hippocampal primary neurons (Fig. 2b).

To investigate the potential regulatory effects of lncRNA$\mathrm{U} 1$ on $N P B W R 1$ gene expression, the full transcripts of lncRNA-U1, the lncRNA- $\mathrm{U}_{66}$, and the $\mathrm{U} 1$ reverse complement (U1R) were cloned for delivery into primary rat neurons using adenovirus. Consistent with results in response to Tat, qRT-PCR, and immunoblotting analyses confirmed increased protein expression of NPBWR1 after neurons were transduced with Ad-U1 (Fig. 2c, d). The upregulation of NPBWR1 in response to Ad-Tat or Ad-U1 


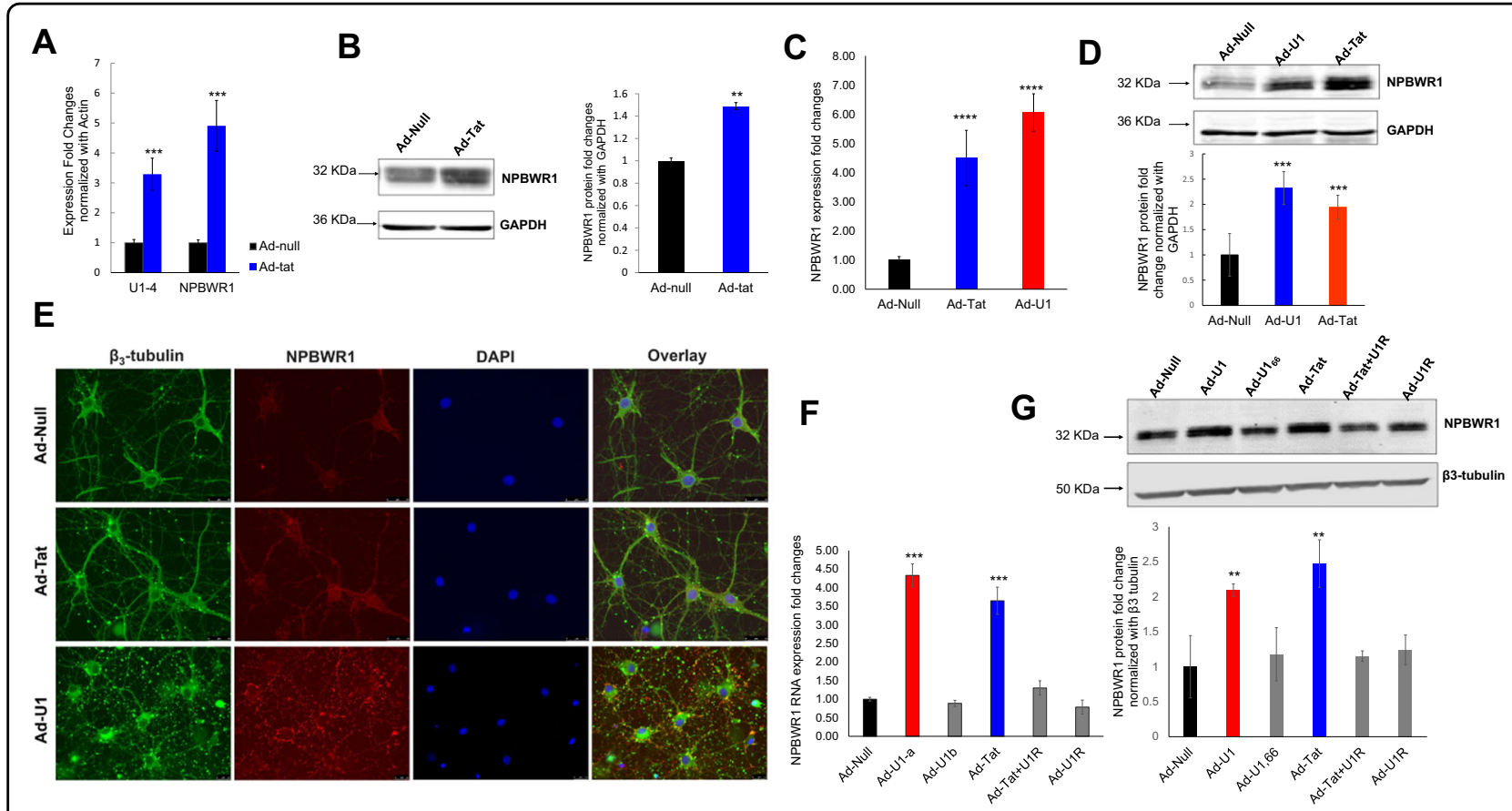

Fig. 2 NPBWR1 expression changes. a The representative bar chart for the results of the GPCR analysis showed the increased expression of NPBWR1 in rat primary neurons transduced with Ad-Tat (MOI: 2, $72 \mathrm{~h}$ ). b Immunoblotting showed an increase in NPBWR1 protein upon Tat expression. c qPCR showed upregulation of NPBWR1 in InCRNA-U1 induced primary neurons, independent of Tat expression. qPCR analysis indicated a 2-fold increased expression of NPBWR1 when primary neurons were transduced with Adeno-U1 (MOI:2) for $72 \mathrm{~h}$. $\mathbf{d}$ Immunoblotting confirmed the overexpression of NPBWR1 upon IncRNA-U1 up-regulation in primary neurons. e ICC confirmed increased NPBWR1 expression in primary neurons upon Tat/U1 treatment, the result of immunoblotting revealed the formation of protein aggregates in the cell borders upon manipulating IncRNA-U1 expression in neurons. $\mathbf{f} \mathrm{q}-\mathrm{PCR}$ analysis compared the NPBWR1 expression in neurons transduced with Ad-U1, Ad-U1 ${ }_{66}$, Ad-U1R as well as Tat and cotransduction of Tat and U1R revealing a correlation between IncRNA-U1 overexpression and changes in NPBWR1 expression. U1 66 splice variant did not, however, alter NPBWR1 expression. $\mathbf{g}$ immunoblotting analysis showed no IncRNA-U1 minor splice variant and confirmed the results from q-PCR analysis.

was also validated via immunocytochemistry (ICC) (Fig. 2e). Notably, these data not only confirmed the increased expression of NPBWR1, but also indicated that IncRNA-U1 overexpression alters the distribution of NPBWR1 from a non-aggregated cytosolic form to condensed aggregates within the neuronal membrane (Fig. 2e). However, $\mathrm{U} 1_{66}$ had no effect on NPBWR1 RNA or protein levels (Fig. 2f, g). In addition, when lncRNA-U1 expression in neurons was knocked down via co-transduction with both Ad-Tat and Ad-U1R, expression levels of NPBWR1 RNA and protein showed no significant increase (Fig. 2f, g). Consistent with the effect of Tat, these results showed that lncRNA-U1 can directly increase the expression of NPBWR1. Furthermore, knock down of IncRNA-U1 via U1R inhibited Tat's capacity to increase expression of NPBWR1. Taken together, these results demonstrated the potential regulatory role of IncRNA-U1 on the expression of NPBWR1 gene.

Although the precise role of NPBWR1 is unknown in vivo, it has been shown to play a role in neuronal homeostasis and metabolism in vitro ${ }^{31,42}$. Specifically, NPBWR1 contributes to cellular responses to hormones and neurotransmitters, and in generating $\mathrm{Ca}^{2+43}$.
IncRNA-U1 modulates neuronal homeostasis by inducing $\mathrm{Ca}^{2+}$ flux

Given the capacity for lncRNA-U1 to alter NPBWR1 expression, along with NPBWR1's proposed role in calcium regulation, we next investigated whether lncRNAU1-mediated increased expression of NPBWR1 led to perturbation in cytosolic $\mathrm{Ca}^{2+}$ transients. To measure $\mathrm{Ca}^{2+}$ concentration in neurons, the green-fluorescent calcium indicator, Fluo-4, was utilized with live-cell microscopy. A sharp increase in the levels of cytosolic $\left[\mathrm{Ca}^{2+}\right]_{c}$ was observed in rat primary neurons transduced with either Ad-Tat or Ad-U1 (Fig. 3a). To assess whether mitochondria respond to the increase in $\left[\mathrm{Ca}^{2+}\right]_{\mathrm{c}}$, we measured mitochondrial $\mathrm{Ca}^{2+}$ uptake. Neurons transduced with Ad-Tat or Ad-U1 showed significant increases in mitochondrial calcium uptake $\left[\mathrm{Ca}^{2+}\right]_{\mathrm{m}}$ in response to $10 \mu \mathrm{M}$ $\mathrm{Ca}^{2+}$ (Fig. 3b-e). Data also revealed that the mitochondrial membrane potential $\left(\Delta \Psi_{\mathrm{m}}\right)$ was collapsed (Fig. 3f) and the level of ATP was significantly declined (Fig. 3g) in response to Ad-Tat or Ad-U1.

Although the mitochondrial calcium uniporter (MCU) mediates calcium transport into mitochondria, reports 


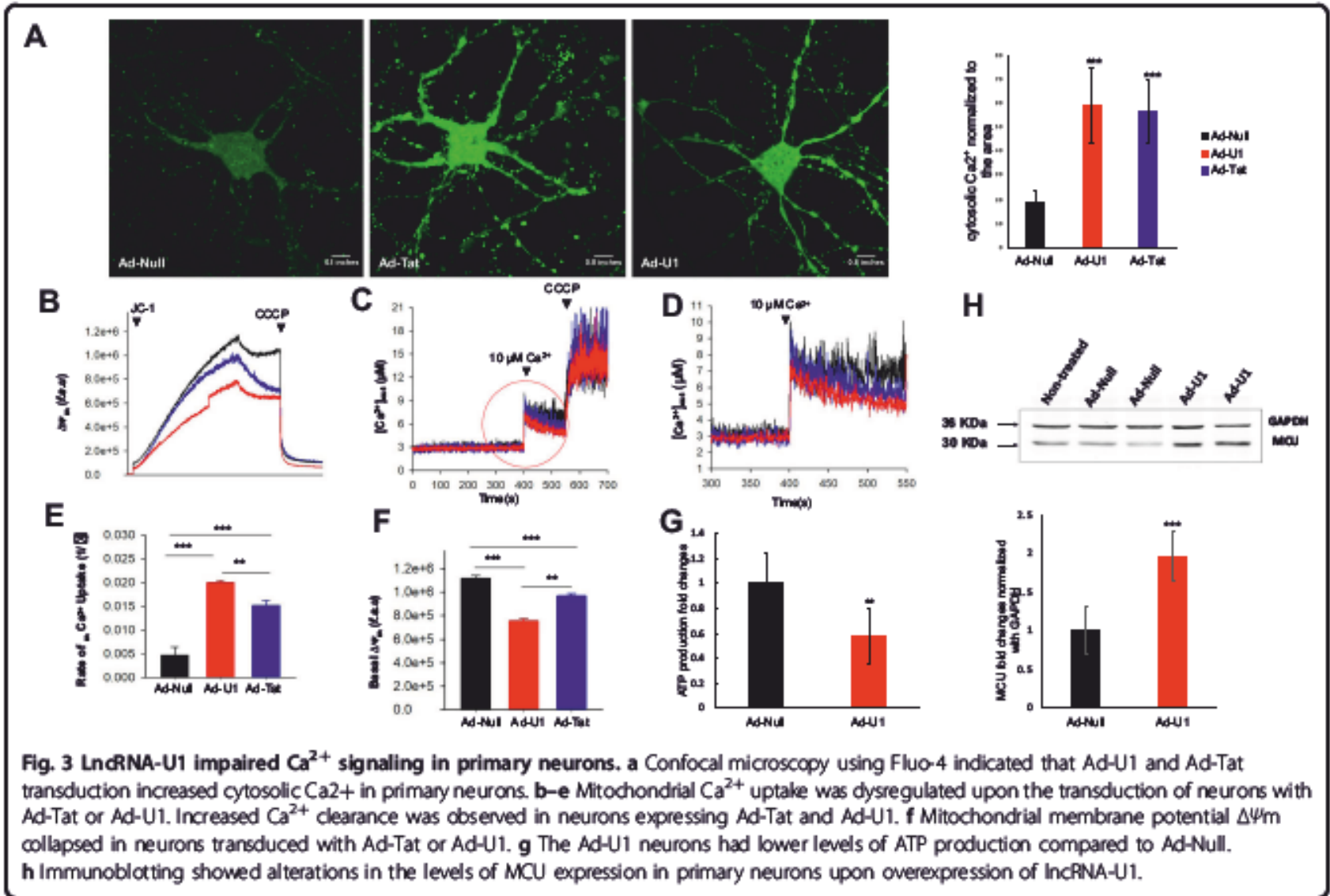

suggest $\mathrm{MCU}$-mediated $\mathrm{Ca}^{2+}$ uptake might result in elevated mitochondrial ROS (mROS) ${ }^{4-46}$. To assess the potential for $\mathrm{MCU}$ to mediate mitochondrial $\mathrm{Ca}^{2+}$ uptake that was observed in response to IncRNA-U1, we investigated changes in mROS production. Accordingly, AdTat/Ad-U1 transduced neurons were incubated with Mitosox red reagent and monitored using confocal microscopy for superoxide production in the mitochondrial matrix. Our analyses indicated that the production of mROS in the mitochondria increased significantly in AdTat and Ad-U1 transduced neurons. Specifically, we observed superoxide accumulation in the neuronal processes in both Ad-Tat and Ad-U1 transduced neurons (Fig. 4a). Moreover, western analysis confirmed the increased expression of $\mathrm{MCU}$ in response to Ad-U1 (Fig. 3h).

Having demonstrated increased mROS and a dynamic shift in calcium from the cytoplasm $\left[\mathrm{Ca}^{2+}\right]_{\mathrm{c}}$ to the mitochondria $\left[\mathrm{Ca}^{2+}\right]_{\mathrm{m}}$, we next evaluated six different mitochondrial parameters: mitochondrial $\mathrm{O}_{2}$ reduction rate (basal oxygen consumption rate (OCR)), nonmitochondrial $\mathrm{O}_{2}$ reduction rate, proton leak, ATP production, maximal respiration, and spare respiratory capacity using the XF96 respiration analysis. In both Ad-Tat and Ad-U1 transduced neurons, significant reductions in basal OCR were noted (Fig. 4b); in addition, blocking proton channels and inhibiting ATP synthesis by oligomycin treatment revealed significant decreases in ATP coupled respiration (Fig, 4c). Similarly, uncoupling oxidative phosphorylation via FCCP indicated maximal OCR was significantly impaired following expression of Tat or IncRNA-U1, while disruption of the electron transport chain with rotenone confirmed these observed alterations in metabolic activity were due to variations in mitochondrial respiration specifically (Fig, $4 d-h$ ). Moreover, analyses of Ad-Null transduced samples confirmed the validity of Tat or U1 induced mitochondrial alterations. Overall, findings suggest that IncRNA-U1 overexpression can induce $\mathrm{Ca}^{2+}$ flood in neuronal mitochondria that could be mediated by MCU and are associated with increased mROS. In turn, IncRNA-U1-dependent mitochondrial dysregulation inhibits multiple bioenergetic mechanisms crucial for neuronal homeostasis.

\section{LncRNA-U1 induces neuronal death via induction of autophagic and apoptotic pathways}

To determine the downstream consequences of IncRNA-U1, cell viability in response to increased IncRNA-U1 was assessed. Initial results using the SYTOX Green assay showed a significant increase in cell death in 


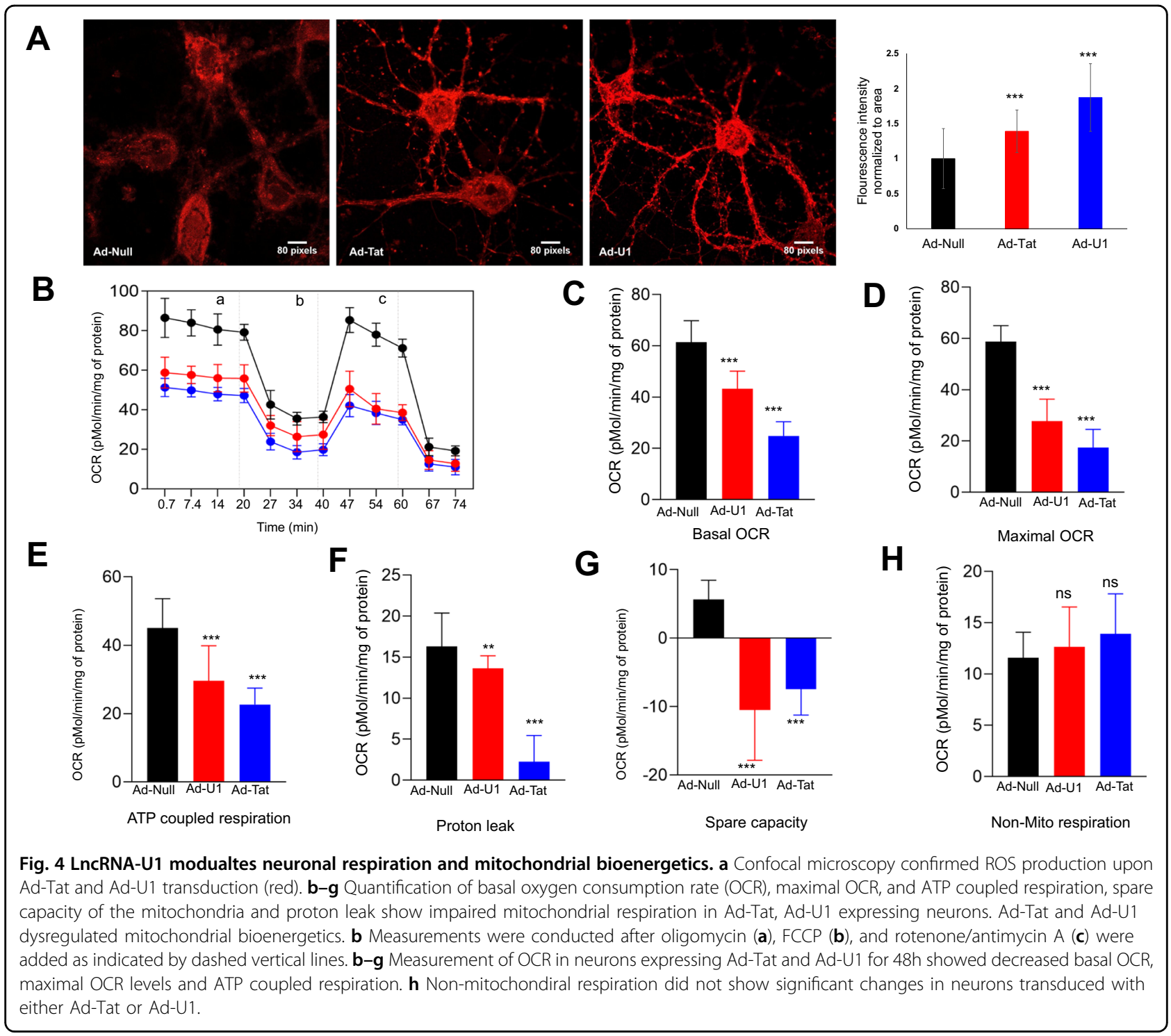

neurons transduced with Ad-U1 (Fig. 5a). In addition, western blot analysis confirmed the activation of the autophagy pathway as indicated by increased LC3-II and decreased p62 in neurons transduced with Ad-U1, and to a lesser degree Ad-Tat (Fig. 5b). Moreover, increased levels pro-apoptotic proteins (i.e. cleaved caspase-3) and decreased anti-apoptotic proteins, (i.e. Bcl-2 and BAG3) were observed in neurons transduced with Ad-U1 or AdTat (Fig. 5c). Taken together, these data suggested lncRNA-U1 induced neuronal death by both autophagic and apoptotic pathways.

\section{In vivo validation of IncRNA-U1 expression in Tat transgenic mice}

To determine if the lncRNA-U1 is a species-specific or a conserved murine lncRNA, we assessed its expression in HIV-1 Tat transgenic mice. RT-PCR analysis of brain tissue from doxycycline (Dox)-inducible GFAP promoterdriven HIV-1 Tat transgenic mice ${ }^{47}$. First, lncRNA-U1 was amplified using the same primer sets for the rat sequence. The RT-PCR amplified fragment was then cloned and sequenced (Fig. 6a) indicating 100\% similarity between mouse and rat sequences (Fig. S3). BLAST analyses of the amplified sequence in the NCBI database calculated an $80 \%$ similarity between lncRNA-U1 and Mus musculus chromosome 1, clone RP23-309D5 sequence $^{48}$. After confirming the expression of lncRNA$\mathrm{U} 1$ in the mouse, total RNA from four different brain regions (hippocampus, prefrontal cortex, cerebellum, and brain stem) of Tat-mice were isolated and utilized for cDNA synthesis. qRT-PCR analyses on the four different Tat transgenic mice treated with DOX versus no DOX confirmed the overexpression of lncRNA-U1 in Tat expressing mice (Fig. 6b). Interestingly, our results 

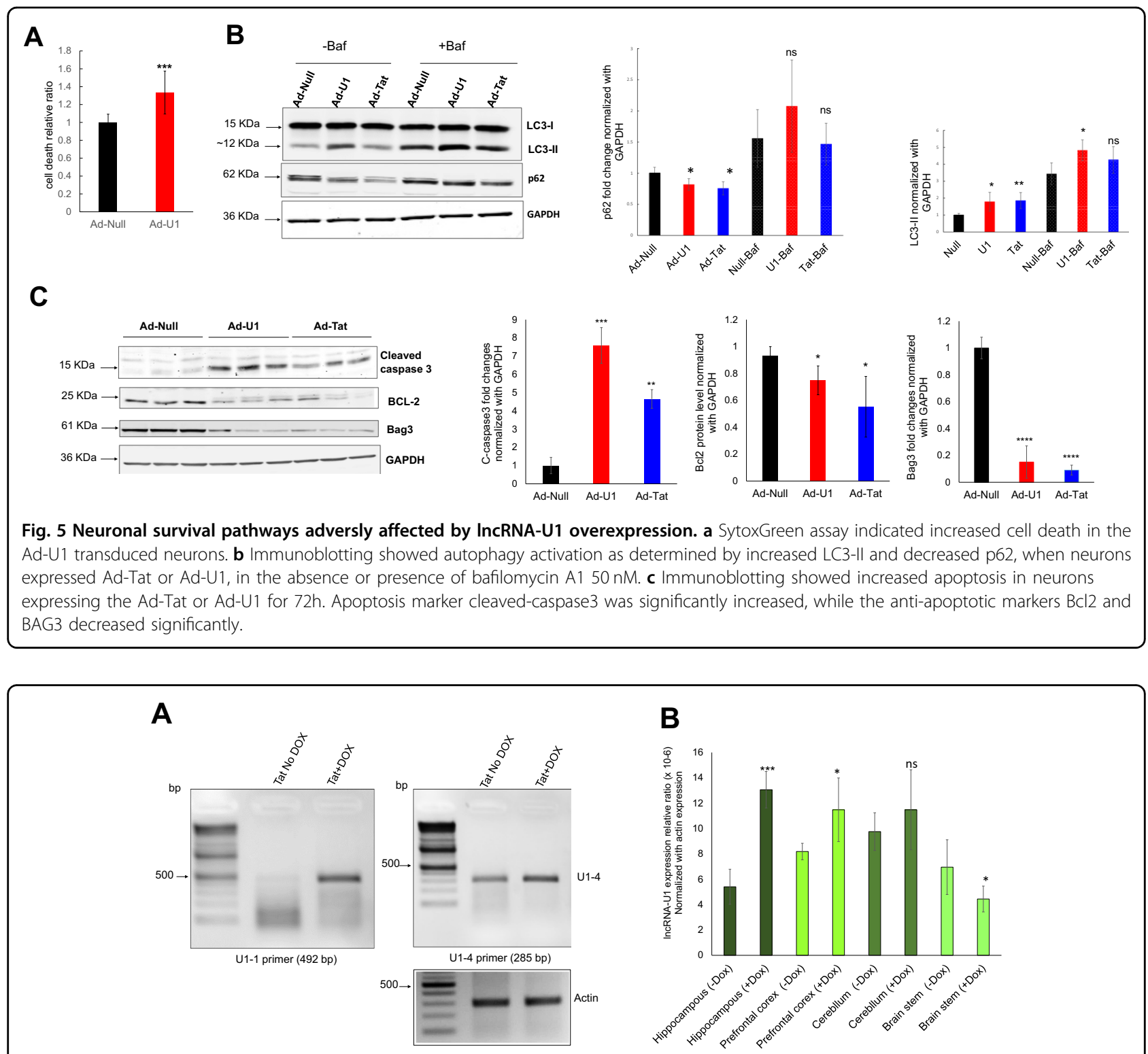

Fig. 6 IncRNA-U1 is upregulated in doxycycline-induced Tat transgenic mice. a RT-PCR confirmed expression alterations of IncRNA-U1 in the Tat expressing mice. $\mathbf{b}$ q-RT PCR analysis confirmed increased expression of IncRNA-U1 in three different brain regions (hippocampus, prefrontal cortex, cerebellum) of Tat expressing transgenic mice, the result indicated more significant increases in hippocampus compared to other brain regions. No changes were observed in the brain stem.

revealed that expression levels of lncRNA-U1 were increased in hippocampus and cortex, while we could not determine significant alteration on lncRNA-U1 in the cerebellum. The IncRNA-U1 expression was downregulated in the brain stem in the presence of Tat. Remarkably, the elevated expression of lncRNA-U1 was greatest in hippocampus followed by the cortex (Fig. 6b).

\section{Discussion}

Given its potential contribution to HIV neuropathogenesis and HAND, it is increasingly relevant to determine the
Tat's capacity to alter neuronal lncRNAs as well as the functional consequences of such alterations on neuronal homeostasis. Findings illustrated a Tat-induced elevation of lncRNA LOC102549805 (lncRNA-U1), along with its splicing variant (lncRNA-U1 $1_{66}$ ). Although increased coexpression of a nearby gene NPBWR1 was also observed in response to Tat, subsequent experiments suggested this pattern of expression might be directly mediated by IncRNA-U1. Furthermore, results suggest that Tat may protect IncRNA-U1 from degradation, provided that increased levels of lncRNA-U1 were observed following 
transcriptional inhibition. Interestingly, elevated levels of lncRNA-U1 were observed in neurons expressing Tat-86 or full-length Tat (101), but not in response to Tat-36 or Tat-72, suggesting U1 variation is specific to full-length Tat proteins. Furthermore, increased lncRNA-U1 induced the accumulation of mitochondrial $\mathrm{Ca}^{2+}$, potentially modulated by $\mathrm{MCU}$, resulting in mitochondrial dysfunction, as indicated by increased mROS, depleted mitochondrial membrane potential and decreased ATP production. Furthermore, the adverse consequences of lncRNA-U1 were associated with decreased neuronal viability, as well as the activation of both autophagy and apoptotic pathways. Thus, our results identified lncRNAU1 as a novel downstream target for Tat, and demonstrated the consequences of increased IncRNA-U1 in disrupting neuronal homeostasis.

In addition to discovering upregulated expression of IncRNA-U1 in response to HIV-1 Tat, current evidence suggested lncRNA-U1 might also be mediating increased co-expression of the NPBWR1 gene. Similar to the effect of Tat, exogenous overexpression of lncRNA-U1 directly elevated the expression of NPBWR1, while the U1R construct was capable of inhibiting the Tat-induced increase in NPBWR1. NPBWR1, a member of G-protein coupled receptors (GPCRs), has been shown to induce $\mathrm{Ca}^{2+}$ mobilization from the cytosol to mitochondria ${ }^{49}$. In line with its possible role, our data confirmed an elevated level of $\left[\mathrm{Ca}^{2+}\right]_{\mathrm{c}}$ transition to $\left[\mathrm{Ca}^{2+}\right]_{\mathrm{m}}$. Results also indicated that this transition could be due in part to MCU, an inward rectifying channel that has a high $\mathrm{Ca}^{2+}$ - carrying capacity that has been suggested to drive mROS production in response to variations in calcium ${ }^{44-46,50}$. Consistent with this finding, immunoassays demonstrated elevated MCU protein and increased mROS. Thus, data suggest that lncRNA-U1-dependent increased expression of NPBWR1 is associated with variation in the distribution of calcium, with transfer from cytosol to mitochondria potentially modulated by MCU. Our studies highlight the effects of Tat on lncRNA-U1 and indicate novel crosstalk between lncRNAs and GPCRs.

Findings also revealed the functional consequences of lncRNA-U1-mediated dysregulation of calcium. In this context, HIV-1 Tat protein has been shown to alter mitochondrial membrane potential and generate ROS, potentially through alterations in $\mathrm{MCU}^{51}$. Moreover, $\mathrm{Ca}^{2+}$ accumulation in mitochondria has been shown to impair numerous aspects of neuronal homeostasis, ranging from regulation of ATP production to cell survival pathways ${ }^{52-54}$. Accordingly, our observations revealed that exogenous overexpression of lncRNA-U1 in rat primary neurons modulated basal and maximal oxygen consumption rate, ATP coupled respiration and spare respiratory capacity. In addition, lncRNA-U1 impaired mitochondrial membrane potential and induced significant elevations in mROS.

Thus, our results suggest a novel mechanism by which Tat stimulates neurotoxicity via elevations in lncRNA-U1 causing an accumulation of mitochondrial calcium, potentially due to activation of NPBWR1 and MCU (Fig. 7). This process is associated with impaired bioenergetic pathways, as well as depleted mitochondrial
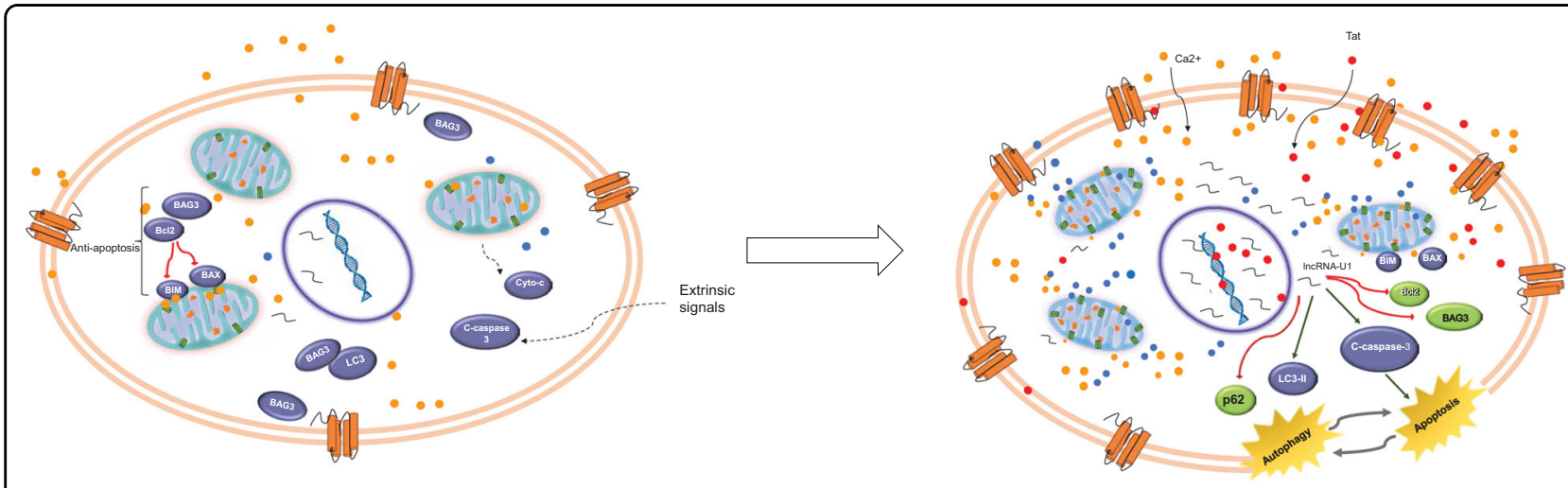

Neuronal normal condition

After Tat expression

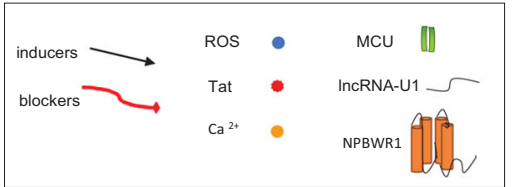

Fig. 7 Schematic showing the hypothetical downstream effects of IncRNA-U1 overexpression in primary hippocampal neurons. a Schematic representation of normal signaling in neurons before the increased expression of InCRNA-U1. b Increased IncRNA-U1 expression alters the neuronal environment by changing the abundance of NPBWR1 followed by increased mitochondrial $\mathrm{Ca}^{2+}$ due to increased MCU channel expression. Ultimately, neuronal homeostasis is altered leading to the activation of both apoptosis and autophagy pathways. 
membrane potential and the elevation in mROS. As a result, imbalances in autophagy and apoptotic pathways ensue, subsequently contributing to compromised neuronal viability.

Consistent with this proposed model, we observed distinct alterations in proteins implicated in autophagy and apoptosis, as well as decreased viability. In particular, lncRNA-U1 significantly decreased BAG3 and BCL-2. This phenomenon is also in consistence with our earlier observation of Tat-induced BAG3 supression ${ }^{55}$. BAG3 is a molecular co-chaperone protein participates in apoptotic inhibition by binding to BCL-2 and forming an antiapoptotic complex ${ }^{56,57}$. Conversely, lncRNA-U1 increased cleaved caspase 3 , a protein critical for apoptosis ${ }^{58}$. Meanwhile, elevations in LC3-II, crucial modulators of autophagy were observed ${ }^{59}$

Based on the current findings, we conclude that Tatmediated induction of lncRNA-U1 results in a series of consequences that significantly compromise neuronal homeostasis. Thus, we provide evidence for a novel mechanism of Tat-induced neurotoxicity. Results illustrated that Tat-mediated lncRNA-U1 increase elevate the co-expression of NPBWR1, a GCPR that plays a crucial role in neuronal homeostasis and metabolism by altering $\mathrm{Ca}^{2+}$ signaling in neurons. In addition, lncRNAU1 was found to disrupt neuronal calcium distribution, potentially due to NPWR1 activation, characterized by calcium accumulation in mitochondria, potentially modulated by MCU. Such calcium dysregulation was associated with compromised mitochondrial functioning, impaired bioenergetic pathways as well as the generation of mROS. Consequently, cell viability was significantly impaired. Taken together, these results warrant further examination of the role of IncRNAs, particularly of lncRNA-U1, in mediating the neuropathological processes of HIV. Moreover, understanding these newly discovered pathways could provide novel therapeutic targets for $\mathrm{PWH}$, including those suffering from HAND.

\section{Materials and methods}

\section{Ethics statement}

All protocols followed the guidelines for the use of laboratory animals and were approved by the Institutional Animal Care and Usage Committee at Temple University.

This study used brain tissue of doxycycline induced GFAP promoter-driven HIV-1 Tat transgenic mice generously provided by Dr. Johnny He to the Comprehensive NeuroAIDS Center ${ }^{47}$. Briefly, mice were divided into two groups, (+DOX) and (-DOX) with 4 animals per group. The doxycycline-induced Tat (iTat) animals were injected i.p. with doxycycline hyclate (DOX) (Sigma-Aldrich) at the dosage of $80 \mathrm{mg} / \mathrm{kg} /$ day for seven days. The control group was injected with saline $(.09 \% \mathrm{NaCl})$ in the same manner as their Dox treated counterparts. After seven days, both groups of mice were sacrificed and brains were extracted/dissected into regions of interest (i.e. hippocampus, cerebellum, brain stem, and frontal cortex $)^{55}$. Total RNA was isolated for each brain region using Trizole reagent (Invitrogen, ThermoFisher, Carlsbad, CA), as previously described ${ }^{55}$.

\section{Rat embryonic cell culture and treatments}

Embryonic rat neurons were cultured as described previously ${ }^{60}$. Briefly, the hippocampi were dissected from E18 rat embryos in cold DPBS (Invitrogen). Tissues were then digested using $0.25 \%$ trypsin and $1 \%$ DNase, at $37{ }^{\circ} \mathrm{C}$ for $17 \mathrm{~min}$. The digested tissues were dissociated in warm Neurobasal (Invitrogen) complete medium containing 2\% serum, 2\% B-27 (Invitrogen), two mM Glutamax (Invitrogen) and $100 \mathrm{U} / \mathrm{ml}$ penicillin and $100 \mathrm{U} / \mathrm{ml}$ streptomycin. The cells were plated at a density of $2 \times 10^{6}$ per well onto $20 \mu \mathrm{g} / \mathrm{ml}$ poly-d-lysine (PK; Sigma), and $1.2 \mathrm{mg} /$ $\mathrm{mL}$ Laminin (Gibco, Thermo Fisher, Carlsbad, CA) coated six-well plates. The media was changed to complete media without serum $24 \mathrm{~h}$ after plating. The media was refreshed twice a week for 10 days by replacing $40 \%$ of the medium from each well. For treatment, neurons were exposed to HIV-1 Tat protein at $50 \mathrm{ng} / \mathrm{ml}$ (101, Immunodiagnostics Inc, Woburn, MA), every $24 \mathrm{~h}$ for $48 \mathrm{~h}$. To transduce neurons with viral constructs including AdNull, Ad-Tat (101 a.a), Ad-U1, Ad-U1 ${ }_{66}$, and Ad-U1R, neurons were incubated for $72 \mathrm{~h}$. To produce viral constructs for transduction, cDNA was cloned from HIV strain 89.6 or amplified lncRNA-U1 into compatible restriction sites of the shuttle plasmid pDC515(IO) and rescued by co-transfecting with pBHGfrtDeltaE1,E3FLP in 293 IQ cells (Microbix Corporation, Mississauga, Ontario Canada). The AD was plaque purified, amplified, and then purified on cesium chloride $(\mathrm{CsCl})$ gradient centrifugation. Plaque purified virus was dialyzed against high salt and $\mathrm{MgCl} 2$ for further purification, since $\mathrm{CsCl}$ does not generate entirely pure preparations. Collected viral particles diluted for concentration measurement at OD260 and the virus concentration calculated based on the number of particles $/ \mathrm{ml}$.

The Ad-U1 $1_{66}$ and Ad-U1R constructs were prepared and verified by PCR amplification. The Ad- $\mathrm{U} 1_{66}$ and AdU1R full transcripts were cloned into pAdenoG plasmid and packaged as adenoviral vector by Applied Biological Materials, Inc. (Richmond, BC, Canada).

Following treatment, total RNA was extracted using Trizol (ThermoFisher, Carlsbad, CA) extraction protocol followed by RNA cleaning protocol using Direct-zol ${ }^{\mathrm{TM}}$ RNA MiniPrep Plus (Zymo Research, Irvine, CA, USA) according to the manufacturer's protocol. Total RNA sequencing ( 80 million reads, $2 \times 75 \mathrm{PE}$ ) was performed by Applied Biological Materials Inc. The RNA-seq 
analysis was performed as described previously ${ }^{33}$. Briefly, version 6.0 annotation of Rattus norvegicus (Rnor 6.0) was applied to identify all non-coding RNAs, including microRNAs and lncRNAs. The $\log _{2}$ of RPKM (Reads Per Kilobase per Million mapped reads) was used to calculate fold changes for the Tat treated neurons versus untreated control neurons. The full gene and transcript sequences for the selected list of IncRNAs were downloaded from the rat genome database (RGD; http://rgd.mcw.edu $)^{34}$.

To measure the decay rate of IncRNA-U1 in the presence or absence of Tat, primary neurons were transduced with either Ad-Null, Ad-Tat or Ad-U1 for fortyeight hours, and subsequently treated with actinomycin $D$ (Sigma, St. Louis, MO) $(2 \mu \mathrm{M})$ to inhibit transcription over the course of eight hours. After 8,4 , and $2 \mathrm{~h}$ of treatment with actinomycin D, total RNA was extracted and utilized for ensuing qPCR analysis. To determine the rate of decay, IncRNA-U1 expression was normalized to its basal expression in non-treated controls.

The Tat mutant $(36,72,86)$ plasmids were provided by our laboratory resources (Comprehensive NeuroAIDS Center). In brief, each mutant was amplified using plasmid specific primers after cloning to pDC515(IO) plasmid. 293-Hek cells were then transduced with Ad-Tat (Tat-101) and transfected with of each mutant (Lipofectamine 3000; Sigma; NY, USA). Forty-eight h after transfection, cells were lysed and total RNA was extracted for subsequent cDNA synthesis and qPCR analysis.

\section{Q-PCR validation of selected IncRNAs}

cDNA synthesis from total RNA was performed with High Capacity cDNA Reverse Transcription Kit (Thermo Fisher Scientific, Burlington, ONT, CANADA) according to the manufacturer's protocol. Primers were designed considering the position of exon junctions in the genomic DNA, primer specificity and efficiency were checked by RT-PCR using Q5 High-Fidelity PCR Kit (New England Biolabs, Ipswich, MA). All qPCR reactions were performed with the LightCycler ${ }^{\circledR} 96$ (Roche, Baltics, UAB) with either the Luna Universal qPCR Master Mix kit (New England Biolabs, Ipswich, MA) or SYBR ${ }^{\mathrm{TM}}$ Green master mix (Applied Biosystems, ThermoFisher), according to manufacturer's protocol. Relative quantification was performed using actin primer reference gene normalization. Primer sequences for amplifying different amplicons of lncRNA-U1 and reference genes are shown in Table S4.

\section{Gene cloning and sequencing}

To confirm the accuracy of amplified fragments using designed primers, RT-PCR products for each primer was separated on $2 \%$ agarose gel and the fragments were extracted using the QIAquick Gel Extraction Kit (QIAGEN, Germantown, MD) and were cloned in Invitrogen One Shot Top 10 chemically component cells. Plasmids were extracted via QIAGEN miniprep kit and submitted for Sanger sequencing (GenWise).

\section{Isolation of protein and western blot analysis}

Neurons in culture were washed with PBS twice and lysed in RIPA buffer (50 mM Tris-HCl, pH 7.5, $150 \mathrm{mM}$ $\mathrm{NaCl}, 0.5 \%$ NP40, 1:100 protease inhibitor cocktail (Calbiochem, San Diego, CA), plus mammalian protease inhibitor cocktail (Sigma-Aldrich, MO, USA). The protein concentration was determined by the Bradford method (BioWorld, Columbus, OH). Equal amounts of proteins were separated on SDS-PAGE and transferred to Odyssey nitrocellulose membrane (Li-Cor, Lincoln, NE) by wet transfer (Bio-Rad, Philadelphia, PA). The following primary antibodies were used for western blotting: antiGPCR GPR7 antibody for NPBWR1 detection (1:1,000, Abcam, Cambridge, MA), anti-GAPDH (1:2,000, Santa Cruz, sc-32233), anti-BCL-2 antibody (1:1000, Santa Cruz, sc-7382), anti-BAG3 antibody (1:1000, Proteintech, 10599-1-AP), anti-p62 antibody (1:1000, Proteintech, 18420-I-AP), anti-LC3 antibody (1:1000, Cell signaling, 3868), anti- $\$ 3$ tubulin antibody (1:1000, Santa Cruz,), anti-cleaved-caspase- 3 antibody (1:1000, Cell signaling, 9661), anti-MCU (1:1000, abcam, ab121499).

\section{Immunolabeling and microscopy}

Primary neurons in two-well chamber slides were washed with PBS and fixed in 4\% paraformaldehyde for 15 min and blocked in 5\% BSA in PBST. The neurons were probed with the following antibodies anti-GPCR GPR7 (1:200) (1:1,000, Abcam, Cambridge, MA), and anti- $\beta 3$-tubulin (1:500) overnight at $4{ }^{\circ} \mathrm{C}$ and washed with cold PBST. Alexa Flour ${ }^{\circledR}$ Secondary antibodies donkey anti-mouse IgG 484 and donkey anti-rabbit IgG 568 has been applied for fluorescent labeling (ThermoFisherScientific, Eugene, OR). VECTASHIELD medium (Vector, Laboratories, Burlingame, CA) was used for DAPI labeling and mounting. Leica fluorescent microscope (Leica Microsystems, IL.) was used for imaging.

\section{Simultaneous measurement of $\mathrm{Ca} 2+$ uptake and $\Delta \psi_{\mathrm{m}}$ in the permeabilized cell system}

Primary neurons were washed in $\mathrm{Ca}^{2+}$ free PBS, pH 7.4. Cells $\left(7 \times 10^{6}\right.$ cells $)$ were suspended and permeabilized with $40 \mu \mathrm{g} / \mathrm{ml}$ digitonin in $1.5 \mathrm{ml}$ of intracellular medium (ICM) composed of $120 \mathrm{mM} \mathrm{KCl}, 10 \mathrm{mM} \mathrm{NaCl}, 1 \mathrm{mM}$ $\mathrm{KH}_{2} \mathrm{PO}_{4}, 20 \mathrm{mM}$ HEPES-Tris, $\mathrm{pH} 7.2$ and $2 \mu \mathrm{M}$ thapsigargin to block the SERCA pump. All measurements were performed in the presence of $5 \mathrm{mM}$ succinate. The simultaneous measurement of $\Delta \Psi \mathrm{m}$ and extramitochondrial $\mathrm{Ca}^{2+}\left(\left[\mathrm{Ca}^{2+}\right]\right.$ out $)$ clearance as an indicator of $\left[\mathrm{Ca}^{2+}\right] \mathrm{m}$ uptake was achieved by loading the permeabilized cells with $\mathrm{JC}-1(800 \mathrm{nM})$ and Fura2-FF $(0.5 \mu \mathrm{M})$, respectively. Mitochondrial uncoupler, carbonyl 
cyanide m-chlorophenyl hydrazone (CCCP, $2 \mu \mathrm{M})$ was added as indicated to collapse the mitochondrial membrane potential. Fluorescence was monitored in a multiwavelength excitation dual-wavelength emission fluorimeter (HORIBA Scientific, Piscataway, New Jersy) as described previously ${ }^{61,62}$.

\section{Measurement of spontaneous cytosolic $\mathrm{Ca}^{2+}$ in live neurons}

Embryonic rat neurons were grown on $25 \mathrm{~mm}$ glass coverslips and loaded with Fluo-4/AM (30 min) in loading buffer $2 \%$ BSA (1X salt solution, $2 \%$ BSA, $0.18 \% \mathrm{~m} / \mathrm{w}$ dextrose, $1 \mathrm{mM}$ Hepes/ $\mathrm{NaOH}, 0.05 \mathrm{mM}$ sulfinpyrazone, $0.003 \% \mathrm{v} / \mathrm{v}$ Pluronic acid) ${ }^{63}$. Cytoplasmic $\mathrm{Ca}^{2+}$ was recorded (510 Meta; Carl Zeiss, Inc. Heidelberg, Germany) at 488 excitations using a $63 \times$ oil objective. Images have been quantified and analyzed using Image ${ }^{64}$.

\section{Measurement of mitochondrial oxygen consumption rate}

Rat primary neurons were cultured on 96-well (XF96) microplate at the concentration of $3 \times 10^{4}$ cells per well (Seahorse Bioscience, Illerica, MA) and transduced with Ad-Null, Ad-Tat or Ad-U1. Forty-eight hours after transduction, neurons were subjected to OCR measurement at $37^{\circ} \mathrm{C}$ in an XF96 extracellular flux analyzer (Seahorse Bioscience). The XF96 extracellular flux assay kit was calibrated using calibration solution (Seahorse Bioscience) in a non- $\mathrm{CO}_{2}, 37^{\circ} \mathrm{C}$ incubator overnight, mitochondrial complexes were sequentially inhibited with a: $10 \mu \mathrm{M}$ oligomycin, b: $10 \mu \mathrm{M}$ FCCP, c: $1 \mu \mathrm{M}$ rotenone/ antimycin A. Basal and maximal OCR, ATP coupled respiration, spare capacity and proton leak were normalized to total protein and analyzed.

\section{Mitochondrial superoxide (mROS) measurement}

Twelve-day old neurons grown on $25 \mathrm{~mm}$ coverslips (Fisherbrand, Pittsburg, PA) were transduced with AdNull, Ad-Tat or Ad-U1. Forty-eight hours after treatment, cells were incubated with mitochondrial oxygen free radical indicator MitoSOX red (Life Technologies, Eugene, OR) for $30 \mathrm{~min}$ at $37^{\circ} \mathrm{C}$. Coverslips were mounted for confocal imaging in an open perfusion microincubator (PDMI-2; Harvard Apparatus). Images were obtained at $561 \mathrm{~nm}$ excitation by using a $63 \times$ oil objective of Confocal microscope (810; Carl Zeiss, Inc.).

\section{Cell death assay}

Rat hippocampal neurons were grown in 96-well plates $\left(3 \times 10^{4}\right.$ cells/well). Seventy-two hours after transduction with AdNull, AdTat or AdU1, cell death was measured using the SYTOX Green cell death assay (Invitrogen). Briefly, cells were incubated at $37^{\circ} \mathrm{C}$ for $15 \mathrm{~min}$ and fluorescent emission was assessed at excitation/emission of $504 / 523 \mathrm{~nm}$ via spectrophotometer to determine damaged cells versus live cells.

\section{Acknowledgements}

We wish to thank past and present members of the Department of Neuroscience and Center for Neurovirology for their support, and sharing of ideas and reagents. We also thank Michael Duggan and Cynthia Papaleo for intellectual and editorial assistance. This work was made possible by a grant P01DA037830 awarded by the NIH to K.K.

\section{Conflict of interest}

The authors declare that they have no conflict of interest.

\section{Publisher's note}

Springer Nature remains neutral with regard to jurisdictional claims in published maps and institutional affiliations.

Supplementary Information accompanies this paper at (https://doi.org/ 10.1038/s41419-020-03033-4).

Received: 18 December 2019 Revised: 16 July 2020 Accepted: 17 July 2020 Published online: 08 October 2020

\section{References}

1. Mediouni, S., Garibaldi Marcondes, M. C., Miller, C., McLaughlin, J. P. \& Valente, S. T. The cross-talk of HIV-1 Tat and methamphetamine in HIV-associated neurocognitive disorders. Front. Microbiol. 6, 1164 (2015).

2. Richman, D. D. et al. The challenge of finding a cure for HIV infection. Science 323, 1304-1307 (2009).

3. Deeks, S. G. et al. Towards an HIV cure: a global scientific strategy. Nat. Rev. Immunol. 12, 607 (2012).

4. Kaul, M., Garden, G. A. \& Lipton, S. A. Pathways to neuronal injury and apoptosis in HIV-associated dementia. Nature 410, 988 (2001).

5. Saylor, D. et al. HIV-associated neurocognitive disorder-pathogenesis and prospects for treatment. Nat. Rev. Neurol. 12, 234-248 (2016).

6. Caldwell, R. L., Egan, B. S. \& Shepherd, V. L. HIV-1 Tat represses transcription from the mannose receptor promoter. J. Immunol. 165 7035-7041 (2000).

7. Clark, E., Nava, B. \& Caputi, M. Tat is a multifunctional viral protein that modulates cellular gene expression and functions. Oncotarget 8, 27569 (2017).

8. Banks, W. A., Robinson, S. M. \& Nath, A. Permeability of the blood-brain barrier to HIV-1 Tat. Exp. Neurol. 193, 218-227 (2005).

9. Nath, A. Human immunodeficiency virus (HIV) proteins in neuropathogenesis of HIV dementia. J. Infect. Dis. 186, S193-S198 (2002).

10. Langford, D. et al. Doxycycline-inducible and astrocyte-specific HIV-1 Tat transgenic mice (iTat) as an HIV/neuroAIDS model. J. Neurovirol. 24, 168-179 (2018).

11. Li, W., Li, G., Steiner, J. \& Nath, A. Role of Tat protein in HIV neuropathogenesis. Neurotox. Res. 16, 205-220 (2009).

12. Hategan, A. et al. HIV Tat protein and amyloid- $\beta$ peptide form multifibrillar structures that cause neurotoxicity. Nat. Struct. Mol. Biol. 24, 379-386 (2017).

13. Bagashev, A. \& Sawaya, B. E. Roles and functions of HIV-1 Tat protein in the CNS: an ovenview. Virol. J. 10, 358 (2013).

14. Rahimian, P. \& He, J. J. HIV-1 Tat-shortened neurite outgrowth through regulation of microRNA-132 and its target gene expression. J. neuroinflammation 13, 247 (2016).

15. Reeder, J. E., Kwak, Y.-T., McNamara, R. P., Forst, C. V. \& D'Orso, I. HIV Tat controls RNA Polymerase $\|$ and the epigenetic landscape to transcriptionally reprogram target immune cells. Elife. 4, e08955 (2015).

16. Rice, A. P. The HIV-1 Tat protein: mechanism of action and target for HIV-1 cure strategies. Curr. Pharm. Des. 23, 4098-4102 (2017).

17. Geiss, G. K. et al. Large-scale monitoring of host cell gene expression during HIV-1 infection using CDNA microarrays. Virology 266, 8-16 (2000).

18. König, R. et al. Global analysis of host-pathogen interactions that regulate early-stage HIV-1 replication. Cell 135, 49-60 (2008).

19. Bushman, F. D. et al. Host cell factors in HIV replication: meta-analysis of genome-wide studies. PLoS Pathog. 5, e1000437 (2009).

20. Strebel, K. Virus-host interactions: role of HIV proteins Vif, Tat, and Rev. Aids 17, S25-S34 (2003).

21. Fernandes, J. C. R., Acuña, S. M., Aoki, J. I., Floeter-Winter, L. M. \& Muxel, S. M Long non-coding RNAs in the regulation of gene expression: physiology and disease. Noncoding RNA 5, 17 (2019). 
22. Fortes, P. \& Morris, K. V. Long noncoding RNAs in viral infections. Virus Res. 212, 1-11 (2016).

23. Lazar, D. C., Morris, K. V. \& Saayman, S. M. The emerging role of long noncoding RNAs in HIV infection. Virus Res. 212, 114-126 (2016).

24. Imam, H., Bano, A. S., Patel, P., Holla, P. \& Jameel, S. The IncRNA NRON modulates HIV-1 replication in a NFAT-dependent manner and is differentially regulated by early and late viral proteins. Sci. Rep. 5, 8639 (2015).

25. Rinn, J. L. \& Chang, H. Y. Genome regulation by long noncoding RNAs. Annu. Rev. Biochem. 81, 145-166 (2012).

26. Wang, K. C. \& Chang, H. Y. Molecular mechanisms of long noncoding RNAs. Mol. Cell 43, 904-914 (2011).

27. Barichievy, S., Naidoo, J. \& Mhlanga, M. M. Non-coding RNAs and HIV: viral manipulation of host dark matter to shape the cellular environment. Front. Genet. 6, 108 (2015).

28. Trypsteen, W. et al. Differential expression of IncRNAs during the HIV replication cycle: an underestimated layer in the HIV-host interplay. Sci. Rep. 6 , 36111 (2016).

29. Briggs, J. A., Wolvetang, E. J., Mattick, J. S., Rinn, J. L. \& Barry, G. Mechanisms of long non-coding RNAs in mammalian nervous system development, plasticity, disease, and evolution. Neuron 88, 861-877 (2015).

30. Shi, C., Zhang, L. \& Qin, C. Long non-coding RNAs in brain development, synaptic biology, and Alzheimer's disease. Brain Res. Bull. 132, 160-169 (2017).

31. Sakurai, T. J. F. NPBWR1 and NPBWR2: implications in energy homeostasis, pain, and emotion. Front. Endocrinol. (Lausanne) 4, 23 (2013).

32. Hondo, M., Ishii, M. \& Sakurai, T. in Orphan G Protein-Coupled Receptors and Novel Neuropeptides, 239-256 (Springer, 2008).

33. Ahooyi, T. M. et al. Dysregulation of neuronal cholesterol homeostasis upon exposure to HIV-1 Tat and cocaine revealed by RNA-sequencing. Sci. Rep. 8 , 16300 (2018).

34. Shimoyama, M. et al. The Rat Genome Database 2015: genomic, phenotypic and environmental variations and disease. Nucleic Acids Res. 43, D743-D750 (2014).

35. Anderson, K. M. et al. Transcription of the non-coding RNA upperhand controls Hand2 expression and heart development. Nature 539, 433 (2016).

36. Kopp, F. \& Mendell, J. T. Functional classification and experimental dissection of long noncoding RNAs. Cell 172, 393-407 (2018).

37. Yin, Y. et al. Opposing roles for the IncRNA haunt and its genomic locus in regulating HOXA gene activation during embryonic stem cell differentiation. cell stem cell 16, 504-516 (2015).

38. Kotzin, J. J. et al. The long non-coding RNA Morrbid regulates Bim and shortlived myeloid cell lifespan. Nature 537, 239 (2016).

39. Westra, $\mathrm{H}-$-J. et al. Systematic identification of trans eQTLs as putative drivers of known disease associations. Nat Genet. 45, 1238 (2013).

40. Innocenti, F. et al. Identification, replication, and functional fine-mapping of expression quantitative trait loci in primary human liver tissue. PLoS Genet. 7, e1002078 (2011).

41. Grundberg, E. et al. Mapping cis-and trans-regulatory effects across multiple tissues in twins. Nat Genet. 44, 1084-1089 (2012).

42. Tanaka, $\mathrm{H}$. et al. Characterization of a family of endogenous neuropeptide ligands for the G protein-coupled receptors GPR7 and GPR8. Proc Natl Acad Sci USA. 100, 6251-6256 (2003).

43. Kiselyov, K., Shin, D. M. \& Muallem, S. Signalling specificity in GPCR-dependent Ca2+ signalling. Cell. Signal. 15, 243-253 (2003).

44. Kirichok, Y., Krapivinsky, G. \& Clapham, D. E. The mitochondrial calcium uniporter is a highly selective ion channel. Nature 427, 360 (2004).
45. Mallilankaraman, K. et al. MICU1 is an essential gatekeeper for MCU-mediated mitochondrial Ca2+ uptake that regulates cell survival. Cell 151, 630-644 (2012).

46. Dong, Z. et al. Mitochondrial Ca2+ uniporter is a mitochondrial luminal redox sensor that augments MCU channel activity. Mol. cell 65, 1014-1028 (2017). e1017.

47. Kim, B. O. et al. Neuropathologies in transgenic mice expressing human immunodeficiency virus type 1 Tat protein under the regulation of the astrocyte-specific glial fibrillary acidic protein promoter and doxycycline. Am. J. Pathol. 162, 1693-1707 (2003).

48. Birren, B., et al. Mus musculus chromosome 1, clone RP23-309D5, complete sequence. https://www.ncbi.nlm.nih.gov/nuccore/AC130658.36 (2005).

49. Tanaka, $\mathrm{H}$. et al. Characterization of a family of endogenous neuropeptide ligands for the G protein-coupled receptors GPR7 and GPR8. Proc. Natl Acad. Sci. USA 100, 6251-6256 (2003).

50. Petrungaro, $C$. et al. The Ca2+-dependent release of the Mia40-induced MICU1-MICU2 dimer from MCU regulates mitochondrial Ca2+ uptake. Cell Metab. 22, 721-733 (2015).

51. Thangaraj, A. et al. HIV-1 TAT-mediated microglial activation: role of mitochondrial dysfunction and defective mitophagy. Autophagy 14, 1596-1619 (2018).

52. Murphy, A. N., Fiskum, G. \& Beal, M. F. Mitochondria in neurodegeneration: bioenergetic function in cell life and death. J. Cereb. Blood Flow. Metab. 19, 231-245 (1999).

53. Zhu, L., Yu, X., Ling, S., Brown, R. \& Kuo, T. Mitochondrial Ca2+ homeostasis in the regulation of apoptotic and necrotic cell deaths. Cell calcium 28, 107-117 (2000).

54. Penna, E., Espino, J., De Stefani, D. \& Rizzuto, R. The MCU complex in cell death. Cell Calcium 69, 73-80 (2018).

55. Cotto, B. et al. Cocaine and HIV-1 Tat disrupt cholesterol homeostasis in astrocytes: Implications for HIV-associated neurocognitive disorders in cocaine user patients. Glia 66, 889-902 (2018).

56. Bonelli, P. et al. BAG3 protein regulates stress-induced apoptosis in normal and neoplastic leukocytes. Leukemia 18, 358 (2004).

57. Das, C. K. et al. BAG3 overexpression and cytoprotective autophagy mediate apoptosis resistance in chemoresistant breast cancer cells. Neoplasia 20, 263-279 (2018).

58. Brentnall, M., Rodriguez-Menocal, L., De Guevara, R. L., Cepero, E. \& Boise, L. H. Caspase-9, caspase-3 and caspase-7 have distinct roles during intrinsic apoptosis. BMC cell Biol. 14, 32 (2013).

59. Tanida, I., Ueno, T. \& Kominami, E. In Autophagosome and Phagosome. 77-88 (Springer, 2008).

60. Ahooyi, T. M. et al. Perturbation of synapsins homeostasis through HIV-1 Tatmediated suppression of BAG3 in primary neuronal cells. Cell Death Dis. 10, 473 (2019).

61. Shanmughapriya, S. et al. Ca2+ signals regulate mitochondrial metabolism by stimulating CREB-mediated expression of the mitochondrial $\mathrm{Ca} 2+$ uniporter gene MCU. Sci. Signal. 8, ra23-ra23 (2015).

62. Mallilankaraman, K. et al. MCUR1 is an essential component of mitochondria Ca 2+ uptake that regulates cellular metabolism. Nat. Cell Biol. 14, 1336 (2012).

63. Madesh, M. et al. Selective role for superoxide in InsP3 receptor-mediated mitochondrial dysfunction and endothelial apoptosis. J. Cell Biol. 170, 1079-1090 (2005)

64. Abràmoff, M. D., Magalhães, P. J. \& Ram, S. J. Image processing with ImageJ. Biophotonics Int. 11, 36-42 (2004). 